\title{
Validação interna do questionário de estágio de prontidão para mudança do comportamento alimentar e de atividade física
}

\author{
Internal validation of the stage of change questionnaire for alimentary and physical activity behaviors
}

\author{
Glauco Barnez P. Cattai ${ }^{1}$, Luzia Jaeger Hintze ${ }^{2}$, Nelson Nardo Junior ${ }^{3}$
}

\section{RESUMO}

Objetivo: Traduzir para a língua portuguesa e adaptar o conteúdo do questionário de avaliação dos estágios de prontidão para mudança do comportamento alimentar e de atividade física proposto por Sutton et al para a aplicação em adolescentes, além de verificar a consistência interna e a reprodutibilidade da nova versão do instrumento.

Métodos: A validação de conteúdo foi realizada por um grupo multiprofissional, foi consultado após a tradução e re-tradução do instrumento com o intuito de gerar uma versão coerente, seguida da adaptação cultural realizada por meio de escala Likert, que objetivou analisar a clareza e a relevância, avaliada pelos adolescentes para cada uma das afirmações. A reprodutibilidade e a consistência interna foram analisadas pelo preenchimento do questionário por 32 adolescentes obesos de ambos os sexos, ingressantes em programa de tratamento multiprofissional da obesidade, com idade média de $13,5( \pm 1,6)$ anos e peso médio de $86,1 \mathrm{~kg}$ $( \pm 18,8)$. Para avaliar a consistência interna, aplicou-se o coeficiente Alpha de Cronbach e, para a reprodutibilidade, os coeficientes de correlação de Pearson, Spearman e intraclasse, além do teste $t$.

Resultados: Os resultados revelaram alta reprodutibilidade do instrumento e, no que diz respeito à consistência interna, foram demonstrados bons índices de confiabilidade para todos os domínios, tendo em vista que todos eles apresentaram valores superiores ou bem próximos a 0,70 .

Conclusões: O questionário apresentou boa consistência interna e reprodutibilidade para avaliar o estágio de prontidão de mudança do comportamento alimentar e de atividade física em adolescentes obesos. Contudo, para que a validade externa do instrumento seja confirmada, é oportuno que outros estudos sejam realizados.

Palavras-chave: adolescente; obesidade; comportamento alimentar; atividade motora; estudos de validação.

\section{ABSTRACT}

Objective: To translate into Portuguese the contents of the questionnaire for adaptation assessment of dietary and physical activity changes proposed by Sutton et al, to be used with adolescents; and to verify the internal consistency and reliability of this tool.

Methods: The content validation was accomplished by a multidisciplinary group, which was consulted after the translation and back translation of the instrument in order to generate a coherent version, followed by the cultural adaptation performed using a Likert's scale, from which adolescents assessed clarity and relevance of each statement. The reliability and internal consistency of the tool were assessed by a questionnaire applied to 32 obese adolescents of both genders from a multiprofessional program for obesity treatment. They had, on average, $13.5 \pm 1.59$ years old and $86.1 \pm 18.8 \mathrm{~kg}$. The Cronbach's alpha coefficient was used to assess the internal consistency, as well as Pearson, Spearman and intraclass correlation coefficients. Also the t-test was used to assess reliability.

Results: The results of test and retests revealed the high reliability of the tool. Internal consistency showed good reliability rates for all areas, with all values higher than or near to 0.70 .

Conclusions: The questionnaire presented good internal consistency and reliability in obese adolescents. However, it
Instituição: Universidade Estadual de Maringá (UEM), Maringá, PR, Brasil ${ }^{1}$ Mestre; Integrante do Núcleo de Estudos Multiprofissionais da Obesidade (NEMO), Maringá, PR, Brasil

${ }^{2}$ Mestranda; Integrante do NEMO, Maringá, PR, Brasil

${ }^{3}$ Doutor; Professor do Departamento de Educação Física da UEM; Coordenador do NEMO, Maringá, PR, Brasil
Endereço para correspondência:

Nelson Nardo Junior

Avenida Colombo, 5.790, Zona 7

CEP 87020-900 - Maringá/PR

E-mail: nnjunior@uem.br

Conflito de interesse: nada a declarar.

Recebido em: 26/5/09

Aceito em: 19/10/09 
is appropriate to carry out further studies in order to confirm the external validity of the instrument.

Key-words: adolescent; obesity; feeding behavior; motor activity; validation studies.

\section{Introdução}

O modelo transteorético, também conhecido como modelo dos estágios de prontidão para mudança do comportamento, surgiu no início dos anos $1980^{(1)}$ a partir de uma análise comparativa dos sistemas de mudança do comportamento usados pela psicoterapia para tratar comportamentos viciadores, como o tabagismo.

Esse modelo utiliza a construção de fases de mudança, ou seja, em vez de modificar os comportamentos considerados inadequados de maneira direta e abrupta, a pessoa o faz em etapas, no contexto de um equilíbrio decisional, buscando autoeficácia no processo de mudança. As fases de mudança passam consecutivamente pelos estágios de pré-contemplação, contemplação, preparo, ação e manutenção, incluindo o aspecto temporal e o motivacional para mudança ${ }^{(2)}$. As alterações devem ocorrer na intenção, atitude e comportamento para que o indivíduo passe de uma fase para outra. Assim, em vez de procurar saber o porquê das mudanças, o modelo busca indicar como estas ocorrem, utilizando os estágios e processos de mudança de comportamento como indicadores ${ }^{(3)}$.

Intervenções que utilizam este modelo baseiam-se no conceito de que mudanças de comportamento ocorrerão quando, e somente se, o indivíduo estiver disposto a isso. Entretanto, estratégias devem ser adotadas para que o paciente evolua dentro dos estágios de prontidão para mudança do comportamento, dos não-saudáveis para os saudáveis ${ }^{(2)}$. Ao longo destes anos, o modelo de estágios de prontidão para mudança do comportamento tornou-se objeto de muitos estudos com vistas ao tabagismo ${ }^{(4)}$, alcoolismo ${ }^{(5)}$ e doenças sexualmente transmissíveis ${ }^{(6)}$.

Mais recentemente, o modelo transteorético passou também a ser utilizado no estudo e tratamento de outros comportamentos de risco para a saúde, incluindo aqueles relacionados à alimentação ${ }^{(7-9)}$ e à atividade física ${ }^{(10-13)}$, diretamente associados ao sobrepeso e à obesidade ${ }^{(14-18)}$. A obesidade tem apresentado aumento alarmante em sua prevalência, atingindo vários países do mundo, tanto desenvolvidos como em desenvolvimento ${ }^{(14,15)}$. É causada por múltiplos fatores, sendo mais fortemente associada aos hábitos alimentares e à prática de atividade física ${ }^{(14,17,18)}$. Essa doença era, até pouco tempo, verificada com maior frequência nos adultos, mas passou a afetar também crianças e adolescentes, tornando-a um dos principais desafios da Saúde Pública na atualidade.

A dificuldade encontrada para tratar a obesidade revela a urgente necessidade de prevenir o surgimento de novos casos e aprimorar os modelos de intervenção existentes a fim de minimizar a carga gerada pela doença ${ }^{(17,19)}$. Nesse sentido, o modelo transteorético tem sido utilizado, tanto como forma de avaliação quanto de tratamento, em intervenções voltadas aos estágios de prontidão para mudança do comportamento associado aos fatores etiológicos da obesidade, como os hábitos alimentares ${ }^{(20-22)}$ e a prática regular de atividade física ${ }^{(13,23)}$. Conhecer o estágio de prontidão para mudança é importante para indivíduos que se submeterão a programas de perda de peso, uma vez que podem direcionar a intervenção, sendo esta específica para cada estágio de prontidão.

No entanto, a maioria dos instrumentos que procuram avaliar os estágios de prontidão para a mudança do comportamento alimentar e de atividade física demanda a presença do pesquisador em cada avaliação, o que dificulta a aplicação simultânea em grupos de pessoas e, além disso, geralmente abordam apenas um item específico, por exemplo a prática ou não de atividade física ${ }^{(10)}$. Diferente disso, o instrumento proposto por Sutton $e t$ al ${ }^{(7)}$ facilita a autoadministração e avalia o estágio de prontidão para mudança do comportamento pautado em um conjunto de itens, além de reunir no mesmo questionário diversos comportamentos relacionados ao processo de perda de peso.

Deste modo, com a finalidade de tornar a utilização desse instrumento viável no Brasil e, ao mesmo tempo, ampliar sua aplicação, procurou-se traduzi-lo e adaptá-lo para a língua portuguesa, de modo a possibilitar sua administração em nossa realidade. Para tanto, houve o cuidado de avaliar se, nesse processo, o instrumento manteria a sua validade interna. Diante disso, este estudo objetivou: a) traduzir para a língua portuguesa e adaptar o conteúdo do questionário de avaliação dos estágios de prontidão para mudança do comportamento alimentar e de atividade física, proposto por Sutton $e t$ a $l^{(7)}$, para a aplicação em adolescentes; b) verificar a consistência interna e a reprodutibilidade da nova versão do instrumento.

\section{Métodos}

O processo de adaptação cultural e validação do questionário de estágios de prontidão para mudança do comporta- 
mento alimentar e de atividade física seguiu rigorosamente a metodologia internacional ${ }^{(24)}$ proposta para esse tipo de estudo, passando assim por seis etapas.

$1^{a}$ Etapa. Tradução do questionário por dois professores formados em inglês, um deles não possuía conhecimento do objetivo do estudo e fez a tradução literal, enquanto o outro, ciente do objetivo do estudo, visou à tradução conceitual do questionário. Obtiveram-se, assim, duas versões do questionário em português (versão tradutor 1 e versão tradutor 2).

$2^{a}$ Etapa. Um grupo multiprofissional, formado por dois profissionais de nutrição, dois de educação física e um de psicologia, analisou ambas as versões a fim de criar uma única versão em português do instrumento (Versão $1 \mathrm{em}$ português).

$3^{a}$ Etapa. Nesta fase, foram realizadas duas novas traduções para a língua inglesa, a partir da versão 1 em português, por dois professores de inglês não conhecedores do questionário, gerando duas novas versões em inglês: back translation1 (b1) e back translation2 (b2).

$4^{a}$ Etapa. De posse das duas novas versões em inglês, o grupo multiprofissional foi consultado novamente com o intuito de possibilitar a comparação entre as versões geradas b1 e b2 com a original, para ajustar possíveis discrepâncias entre elas. Para isso, quando necessário, as orações foram reescritas até se tornarem consenso pelo grupo multiprofissional. Assim, gerou-se a Versão 2 em português.

$5^{a}$ Etapa. Foram convidados a participar do estudo todos os adolescentes ingressantes ( $n=32)$ no Programa Multiprofissional de Tratamento da Obesidade (PMTO), desenvolvido na Universidade Estadual de Maringá, em fevereiro de 2008. Essa amostra foi composta por conveniência, pela necessidade da realização de teste e reteste. Os critérios para inclusão no estudo basearam-se na condição de que esses adolescentes estivessem inscritos no PMTO e apresentassem obesidade segundo os pontos de corte propostos por Cole et al ${ }^{(25)}$. O objetivo desta fase era verificar se as questões estavam suficientemente claras e se eram aplicáveis ao contexto brasileiro (relevantes). Para isso, foi solicitado aos adolescentes que atribuíssem notas para cada uma das afirmações quanto à sua clareza e relevância por meio da escala Likert de 5 pontos, na qual zero indicava que a afirmação não estava e/ou não era nem um pouco clara/relevante para o adolescente e 5 , que a afirmação estava e/ou era totalmente clara/relevante. Foram consideradas claras e relevantes (aplicáveis) as questões que atingiram um escore médio superior a 3 . Esse processo foi concluído quando os 32 adolescentes não apresentaram dú- vidas quanto à redação das afirmações do instrumento. Dessa forma, criou-se a versão definitiva do questionário.

$6^{a}$ Etapa. O questionário foi autoadministrado nos mesmos 32 adolescentes obesos, selecionados para participar do PMTO, em dois momentos distintos, com um intervalo de uma semana, seguindo-se rigorosamente os mesmos procedimentos a fim de avaliar a sua reprodutibilidade.

O questionário de avaliação do Estágio de Prontidão para Mudança de Comportamento Alimentar e de Atividade Física, desenvolvido especificamente para pessoas adultas que procuram programas para perda de peso, é composto por 38 afirmações, distribuídas em quatro domínios: tamanho e quantidade das porções; quantidade de gordura na dieta; consumo de frutas e vegetais; prática de atividade física. Esses domínios foram criados a partir de comportamentos que influenciam o controle do peso. As respostas para cada afirmação variam em uma escala Likert que apresenta cinco opções de resposta correspondentes aos estágios de prontidão para mudança do comportamento, conforme segue: 1 (précontemplação); 2 (contemplação); 3 (preparação); 4 (ação); e 5 (manutenção). A versão final do questionário em português está disponível com o autor correpondente.

O cálculo do índice de massa corporal foi realizado por meio da equação $\left[I M C=\right.$ peso $(\mathrm{kg}) /$ estatura $\left.^{2}(\mathrm{~m})\right]$. A aferição do peso foi feita em balança eletrônica com capacidade para $150 \mathrm{~kg}$ e precisão $0,1 \mathrm{~kg}$. A estatura foi mensurada com um estadiômetro de precisão de $0,1 \mathrm{~cm}$ e capacidade para medir até $2 \mathrm{~m}$. As medidas de circunferência foram realizadas com fita métrica inextensível. Como referência para a medida da cintura, foi utilizado o menor perímetro da região abdominal. As medidas de circunferência abdominal e do quadril tiveram como referencial anatômico, respectivamente, a cicatriz umbilical e a maior porção da região glútea.

O tratamento estatístico foi realizado com auxílio do Statistical Package for the Social Sciences versão 14.0. A estatística descritiva empregada para caracterizar a amostra envolveu as medidas de tendência central e dispersão (média e desvio padrão). Para avaliar a consistência interna do instrumento, recorreu-se ao coeficiente Alpha de Cronbach. A reprodutibilidade do instrumento foi verificada por meio dos coeficientes de correlação de Pearson, Spearman e intraclasse, além do teste $t$ de Student.

Os procedimentos utilizados nesta pesquisa acompanharam as regulamentações exigidas pela Resolução 196/96 do Conselho Nacional de Saúde sobre pesquisa envolvendo seres humanos, sendo aprovada pelo Comitê de Ética em Pesquisa da Universidade Estadual de Maringá. Os pais ou responsá- 
Tabela 1 - Distribuição dos valores da média e desvio padrão das características antropométricas e idade dos adolescentes

\begin{tabular}{lccc}
\hline Variáveis & Meninos $(\mathbf{n}=\mathbf{1 2})$ & Meninas $(\mathbf{n}=\mathbf{2 0})$ & Total $(\mathbf{n}=\mathbf{3 2})$ \\
\hline Idade $($ anos $)$ & $13,5 \pm 1,5$ & $13,5 \pm 1,7$ & $13,5 \pm 1,6$ \\
Peso $(\mathrm{kg})$ & $91,0 \pm 15,7$ & $83,4 \pm 20,2$ & $86,1 \pm 18,8$ \\
Estatura $(\mathrm{m})$ & $1,71 \pm 0,09^{*}$ & $1,60 \pm 0,08^{*}$ & $1,64 \pm 0,10$ \\
IMC $\left(\mathrm{kg} / \mathrm{m}^{2}\right)$ & $30,4 \pm 3,6$ & $32,0 \pm 5,4$ & $31,5 \pm 4,9$ \\
Circunferência cintura $(\mathrm{cm})$ & $90,8 \pm 8,7$ & $87,6 \pm 11,8$ & $88,6 \pm 10,9$ \\
Circunferência abdome $(\mathrm{cm})$ & $100,9 \pm 8,3$ & $100,7 \pm 13,7$ & $100,7 \pm 12,0$ \\
Circunferência quadril $(\mathrm{cm})$ & $108,7 \pm 8,6$ & $109,8 \pm 12,9$ & $109,5 \pm 11,5$ \\
RCQ & $0,84 \pm 0,04$ & $0,80 \pm 0,07$ & $0,81 \pm 0,06$ \\
\hline
\end{tabular}

RCQ: relação cintura quadril; IMC: índice de massa corporal. *Teste $t p=0,001$.

veis de cada adolescente incluído no estudo assinaram o termo de consentimento livre e esclarecido e foram assegurados do anonimato das informações.

\section{Resultados}

Dos 32 adolescentes, $62,5 \%$ eram do sexo feminino. Todos eram estudantes, sendo que $84,4 \%$ frequentavam escolas públicas; o nível educacional variou da $5^{a}$ série do ensino fundamental ao $3^{\circ}$ ano do ensino médio. Com relação à classificação econômica, foi utilizado o instrumento proposto pela $\mathrm{ABEP}^{(26)}$, que enquadra as pessoas nas classes $\mathrm{A}$, B, C, D ou E a partir da pontuação alcançada, sendo a classe $\mathrm{A}$ a de maior poder aquisitivo e a classe $\mathrm{E}$ a de menor. Essa classificação indicou que $9,4 \%, 56,2 \%$ e $34,4 \%$ provinham das classes A, B e C, respectivamente. As características antropométricas e de idade dos adolescentes participantes do estudo estão descritas na Tabela 1.

Durante a validação do conteúdo (equivalência cultural) do questionário, apenas quatro afirmações não atingiram escore médio superior a três para relevância. Foram elas as afirmações 6 dos grupos relacionados às porções de alimentos (tamanho/quantidade) e ao consumo de frutas e vegetais, ambas reescritas para corresponder ao contexto brasileiro. As afirmações 4 e 9 do grupo relacionado à atividade física foram complementadas a fim de apresentarem atividades ligadas ao universo dos adolescentes, uma vez que o questionário havia sido originalmente aplicado a adultos de 40 a 69 anos de idade $^{(7)} \mathrm{e}$, portanto, apresentavam atividades normalmente ausentes no cotidiano de adolescentes com idade média de $13,5 \pm 1,6$ anos. No que se refere à clareza das questões, todas apresentaram um escore médio maior que 3, não sendo necessário reformulá-las. Em média, o tempo gasto para preencher o questionário foi de 12 minutos.

$\mathrm{Na}$ Tabela 1, observa-se que apenas a estatura apresentou diferença significativa entre os sexos. Os valores médios das variáveis antropométricas revelam a gravidade da obesidade desses adolescentes tanto pelo Índice de Massa Corporal, que chega a ultrapassar o critério de obesidade estabelecido para adultos ${ }^{(27)}$, como pela circunferência da cintura e pela relação cintura quadril, bem próxima dos pontos de corte estabelecidos para adultos ${ }^{(28)}$.

A reprodutibilidade do instrumento (teste-reteste) foi verificada por meio dos testes t de Student, correlação de Pearson, Spearman e intraclasse, apresentados na Tabela 2. O teste $t$ não apresentou diferença significativa entre os momentos (teste e reteste) em nenhum dos domínios do instrumento. Entretanto, os coeficientes de correlação utilizados demonstraram correlações positivas entre fortes e muito fortes e altamente significativas $(p<0,01)$. Esses resultados refletem a alta reprodutibilidade do instrumento.

A consistência interna do instrumento foi avaliada pelo método Alpha de Cronbach. Os valores totais de cada domínio foram calculados, assim como a variação do Alpha quando eliminados itens (afirmações) de cada domínio, sendo apresentados os valores mínimos e máximos na Tabela 3.

Os resultados encontrados acerca da consistência interna do instrumento demonstram bons índices de confiabilidade para todos os domínios do instrumento, com valores superiores ou bem próximos a 0,70 , corte considerado satisfatório para a maioria dos pesquisadores, segundo Leech $e t a^{(29)}$. Os melhores índices foram obtidos nos domínios 'quantidade de gordura na dieta' $(0,83)$ e 'consumo de frutas e vegetais' $(0,84)$.

\section{Discussão}

A preocupação com a obesidade infanto-juvenil se deve ao fato de que crianças e adolescentes obesos apresentam grande chance de se tornarem adultos obesos, além de mostrarem, em idades muito precoces, uma série de doenças não-transmissíveis associadas à obesidade ${ }^{(20,30)}$. Diante disso, ações 
Tabela 2 - Reprodutibilidade dos domínios do questionário de estágio de prontidão para mudança do comportamento alimentar e de atividade física

\begin{tabular}{lcccc}
\hline \multirow{2}{*}{ Domínios do instrumento } & Teste $\boldsymbol{t}$ & Pearson & Spearman & Intraclasse \\
\cline { 2 - 5 } & Valor $\boldsymbol{t}$ & Valor $\boldsymbol{r}$ & Valor $\boldsymbol{r}_{\mathrm{s}}$ & Valor R \\
\hline Porções (tamanho/quantidade) & $-1,190$ & $0,907^{*}$ & $0,878^{*}$ & $0,904^{*}$ \\
Quantidade de gordura na dieta & $-0,648$ & $0,821^{*}$ & $0,856^{*}$ & $0,815^{*}$ \\
Consumo de frutas e vegetais & $-0,608$ & $0,942^{*}$ & $0,937^{*}$ & $0,940^{*}$ \\
Prática de atividade física & 0,515 & $0,880^{*}$ & $0,880^{*}$ & $0,882^{*}$ \\
\hline
\end{tabular}

${ }^{*}$ Correlação significativa para $p<0,01$.

Tabela 3 - Avaliação da consistência interna do questionário de estágio de prontidão para mudança do comportamento alimentar e de atividade física

\begin{tabular}{lcc}
\hline \multirow{2}{*}{ Domínios } & Alpha de Cronbach & Variação do Alpha se item eliminado \\
\cline { 2 - 3 } & Valor $\alpha$ & Valor $\alpha$ \\
\hline Porções (tamanho/quantidade) & 0,78 & $0,72-0,82$ \\
Quantidade de gordura na dieta & 0,83 & $0,79-0,84$ \\
Consumo de frutas e vegetais & 0,84 & $0,80-0,83$ \\
Prática de atividade física & 0,69 & $0,63-0,69$ \\
\hline
\end{tabular}

que possam contribuir para reduzir essa enfermidade, como aplicar o modelo transteorético, tornam-se fundamentais.

Assim, meios que facilitem a coleta de dados como questionários autoadministrados podem ajudar a aplicar as técnicas acima propostas. O questionário proposto por Sutton et $a l^{(7)}$ é um dos poucos que objetiva mensurar os estágios de prontidão para mudança do comportamento alimentar e de atividade física em pessoas que pretendem reduzir o peso corporal. No entanto, a utilização de um questionário de avaliação deve respeitar o contexto no qual está inserido o avaliado, por isso não é recomendado o seu uso em outro contexto e em outra língua. Nesse sentido, a validação deste instrumento vai além da simples tradução do questionário original, sendo necessário adaptar a linguagem ao contexto brasileiro, aproximando-a da realidade da população em questão.

A avaliação do estágio de prontidão para mudança do comportamento pode contribuir substancialmente para as ações voltadas à promoção da saúde das populações ${ }^{(9,13)}$, sobretudo quando são avaliados os hábitos alimentares e de atividade física, os quais estão entre os principais fatores etiológicos de diversas doenças crônicas não-transmissíveis ${ }^{(16-18)}$. Além da importância de avaliar o estágio de prontidão para mudança do comportamento, o modelo transteorético fornece os elementos necessários para que pesquisadores e profissionais da saúde possam intervir em um grupo de pessoas ${ }^{(21,31)}$ ou individualmente ${ }^{(13)}$ no intuito de favorecer a adoção de uma alimentação adequada e níveis satisfatórios de atividade física, que contribuirão para a redução do peso corporal. Além disso, a utilização deste instrumento por profissionais da saúde poderá permitir avaliar a efetividade de programas de tratamento e campanhas de promoção da saúde, sobretudo daqueles voltados ao tratamento ou prevenção da obesidade na adolescência.

Apesar de o estudo apresentar contribuições importantes para a literatura da área, é preciso considerar que a seleção da amostra não-randomizada, assim como a sua homogeneidade e tamanho, representam limitações que podem ser mais bem controladas em pesquisas futuras. Portanto, não se pretende com este trabalho esgotar a discussão acerca da validade externa do instrumento, uma vez que, de acordo com Sutton $e t a l^{(7)}$, este processo não representa uma tarefa simples ou direta.

Assim, conclui-se que a versão em português do questionário de avaliação do estágio de prontidão para mudança do comportamento dos hábitos alimentares e de atividade física apresentou valores de confiabilidade e validade interna apropriados para a sua aplicação em adolescentes. A facilidade de aplicação deste instrumento possibilita sua utilização em diversos tipos de estudos e/ou tratamentos. Contudo, para que a segurança e a efetividade do instrumento sejam confirmadas, é oportuno que outros estudos sejam realizados. 


\section{Referências bibliográficas}

1. Prochaska JO, DiClemente CC. Stages and processes of self-change in smoking: toward an integrative model of change. J Consult Clin Psychol 1983:51:390-5.

2. Prochaska JO, DiClemente CC. Common processes of change in smoking, weigh control, and psychological distress. In: Shiffman S, Wills T, editors. Coping and substance abuse. San Diego, CA: Academic Press; 1996, p. 345-63.

3. Prochaska JO, Velicer WF. The transtheoretical model of health behavior change. Am J Health Promot 1997;12:38-48.

4. Schumann A, John U, Ulbricht S, Rüge J, Bischof G, Meyer C. Variability of tailoring of a smoking cessation intervention based on the transtheoretical model. Addict Behav 2007;32:3083-7.

5. Callaghan RC, Taylor L, Cunningham JA. Does progressive stage transition mean getting better? A test of the Transtheoretical Model in alcoholism recovery. Addiction 2007;102:1588-96.

6. Horowitz SM. Applying the Transtheoretical Model to pregnancy and STD prevention: a review of the literature. Am J Health Promot 2003;17:304-28.

7. Sutton K, Logue E, Jarjoura D, Baughman K, Smucker W, Capers C. Assessing dietary and exercise stage of change to optimize weight loss interventions. Obes Res 2003;11:641-52.

8. Chang CT. Applicability of the stages of change and Weight Efficacy Lifestyle Questionnaire with natives of Sarawak, Malaysia. Rural and Remote Health [serial on the Internet]. 2007;7:864. [cited 2010 Apr 27]. Available from: http:// www.rrh.org.au.

9. Toral N, Slater B. Transtheoretical model approach in eating behavior. Cienc Saude Coletiva 2007;12:1641-50.

10. Guedes DP, Santos CA, Lopes CC. Stages of behavior change and habitual physical activity in college students. Rev Bras Cineantropom Desempenho Hum 2006;8:5-15.

11. Maddison R, Prapavessis H. Exercise behavior among New Zealand adolescents: a test of the transtheoretical model. Pediatr Exerc Sci 2006;18:351-63.

12. Kosma M, Ellis R, Cardinal BJ, Bauer JJ, McCubbin JA. The mediating role of intention and stages of change in physical activity among adults with physical disabilities: an integrative framework. J Sports Exerc Psychol 2007;29:21-38.

13. Marcus BH, Emmons KM, Simkin-Silverman LR, Linnan LA, Taylor ER, Bock $\mathrm{BC}$ et al. Evaluation of motivationally tailored vs. standard self-help physical activity interventions at the workplace. Am J Health Promot 1998;12:246-53.

14. Bouchard C. Physical activity and obesity. Champaign, IL: Human Kinetcs, 2000.

15. Weinsier RL, Hunter GR, Heini AF, Goran MI, Sell SM. The etiology of obesity: relative contribution of metabolic factors, diet, and physical activity. Am J Med 1998;105:145-50.
16. WHO. Global strategy on diet, physical activity and health. Geneva: World Health Organization; 2004.

17. Monteiro CA. Velhos e novos males da saúde no Brasil: a evolução do país e de suas doenças. $2^{\text {nd }}$ ed. São Paulo: Hucitec; 2000.

18. WHO. Diet, nutrition and the prevention of chronic diseases: report of a joint WHO expert consultation. Report series n. 916. Geneva: World Health Organization; 2003.

19. Sjöström L, Lidroos AK, Peltonen M, Torgerson J, Bouchard C, Carlsson B et al. Lifestyle, diabetes, and cardiovascular risk factors 10 years after bariatric surgery. N Engl J Med 2004;351:2683-93.

20. Hawkins DS, Hornsby PP, Schorling JB. Stages of change and weight loss among rural African American women. Obes Res 2001;9:59-67.

21. Ni Mhurchu C, Margetts BM, Speller VM. Applying the stages-of-change model to dietary change. Nutr Rev 1997;55:10-6.

22. Johnson SS, Paiva AL, Cummins CO, Johnson JL, Dyment SJ, Wright JA et al. Transtheoretical model-based multiple behavior intervention for weight management: effectiveness on a population basis. Prev Med 2008;46: 238-46.

23. Kosma M, Cardinal BJ, McCubbin JA. Predictors of physical activity stage of change among adults with physical disabilities. Am J Health Promot 2004;19:114-7.

24. Beaton D, Bombardier C, Guillemin F, Ferraz MB [homepage on the Internet] Recommendations for the cross-cultural adaptation of the DASH \& QuickDASH outcome measures; 2007 [cited 2010 Apr 27]. Available from: http://www.dash. iwh.on.ca/assets/images/pdfs/X-CulturalAdaptation-2007.pdf

25. Cole TJ, Bellizzi MC, Flegal KM, Dietz WH. Establishing a standart definition for child overweight and obesity worldwide: international survey. BMJ 2000;320:1240-3.

26. Associação Brasileira de Empresas de Pesquisa (ABEP) [homepage on the internet]. São Paulo: Associação Brasileira de Empresas de Pesquisa. [cited 2010 Apr 27]. Available from: http://www.abep.org.

27. WHO. Obesity: preventing and managing the global epidemic. WHO Technical Report Series 894. Geneva: World Health Organization; 1998.

28. Expert panel on the identification, evaluation, and treatment of overweight and obesity in adults. Executive summary of the clinical guidelines on the identification, evaluation, and treatment of overweight and obesity in adults. Arch Intern Med 1998;158:1855-67.

29. Leech NL, Barrett KC, Morgan GA. SPSS for intermediate statistics: use and interpretation. $2^{\text {nd }}$ ed. New Jersey: LEA; 2005.

30. WHO/Europe. The challenge of obesity in the WHO European Region and the strategies for response. Europe: World Health Organization; 2007.

31. Frenn M, Malin S, Bansal NK. Stage-based interventions for low-fat diet with middle school students. J Pediatr Nurs 2003;18:36-45. 